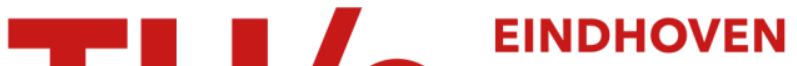

\section{Three-dimensional magnetic field modeling of a cylindrical Halbach Array}

Citation for published version (APA):

Meessen, K. J., Gysen, B. L. J., Paulides, J. J. H., \& Lomonova, E. (2010). Three-dimensional magnetic field modeling of a cylindrical Halbach Array. IEEE Transactions on Magnetics, 46(6), 1733-1736.

https://doi.org/10.1109/TMAG.2010.2042038

DOI:

10.1109/TMAG.2010.2042038

Document status and date:

Published: 01/01/2010

\section{Document Version:}

Publisher's PDF, also known as Version of Record (includes final page, issue and volume numbers)

\section{Please check the document version of this publication:}

- A submitted manuscript is the version of the article upon submission and before peer-review. There can be important differences between the submitted version and the official published version of record. People interested in the research are advised to contact the author for the final version of the publication, or visit the $\mathrm{DOI}$ to the publisher's website.

- The final author version and the galley proof are versions of the publication after peer review.

- The final published version features the final layout of the paper including the volume, issue and page numbers.

Link to publication

\section{General rights}

Copyright and moral rights for the publications made accessible in the public portal are retained by the authors and/or other copyright owners and it is a condition of accessing publications that users recognise and abide by the legal requirements associated with these rights.

- Users may download and print one copy of any publication from the public portal for the purpose of private study or research.

- You may not further distribute the material or use it for any profit-making activity or commercial gain

- You may freely distribute the URL identifying the publication in the public portal.

If the publication is distributed under the terms of Article 25fa of the Dutch Copyright Act, indicated by the "Taverne" license above, please follow below link for the End User Agreement:

www.tue.nl/taverne

Take down policy

If you believe that this document breaches copyright please contact us at:

openaccess@tue.nl

providing details and we will investigate your claim. 


\title{
Three-Dimensional Magnetic Field Modeling of a Cylindrical Halbach Array
}

\author{
Koen J. Meessen, Bart L. J. Gysen, Johannes J. H. Paulides, and Elena A. Lomonova \\ Eindhoven University of Technology, Eindhoven MB 5600, The Netherlands
}

\begin{abstract}
A semi-analytical description of the 3-D magnetic field distribution of a cylindrical quasi-Halbach permanent magnet array is derived. This model avoids the necessity of time-consuming finite element analyses and allows for fast parameterization to investigate the influence of the number of segments on the magnetic flux density distribution. The segmented magnet is used to approximate an ideal radial magnetized ring in a cylindrical quasi-Halbach array. The model is obtained by solving the Maxwell equations using the magnetic scalar potential and describes the magnetic fields by a Fourier series.
\end{abstract}

Index Terms-Actuators, magnetic fields, magnetic scalar potential, modeling, permanent magnets.

\section{INTRODUCTION}

$\mathbf{T}$ HE NEED FOR efficient actuators with high force density in industrial applications is rapidly growing. Permanent magnet (PM) actuators appear to be a good class of machines to fulfill these requirements. Several papers have been written on the subject of the design of PM magnet actuators with various PM configurations. For example, Halbach structures are exploited to achieve an even higher power density with an increased amount of PM material [1]. Due to the evolution of the magnetic materials and production techniques, PMs in various shapes and with different magnetization patterns emerge and the approximation of an ideal Halbach magnetization improves. In spite of all efforts, the price of these magnets at this moment is still high, and hence, the use of magnets with simple shapes and an easy magnetization is preferred. Therefore, by using multiple small magnets with a simple shape, more complex magnetization patterns are approximated.

Regarding tubular permanent magnet actuators (TPMAs), several papers discuss the application of quasi-Halbach magnetization shown in Fig. 1(a), with radial and axial magnetized PMs. However, the radial magnetized ring magnet shown in Fig. 1(b) is difficult to magnetize especially for small radii. Therefore, in practice this PM is often approximated by diametrically magnetized segments as shown in Fig. 1(c). This segmented PM results in a 3-D effect, hence, for the exact magnetic fields in the actuator, a 3-D analysis is required. Previous papers describing tubular actuators with Halbach magnetization consider the 2-D problem with perfect radial magnetized magnets [2], [3].

In this paper a 3-D model is derived which provides the magnetic field expression for segmented quasi-Halbach arrays. To calculate the magnetic fields, a semi-analytical formulation of the magnetic scalar potential in the 3-D cylindrical coordinate system is derived. Although the model is quite complex to derive, it avoids the use of time consuming 3-D Finite element analysis (FEA), and once implemented can easily be used to

Manuscript received October 30, 2009; accepted January 22, 2010. Current version published May 19, 2010. Corresponding author: K. J. Meessen (e-mail: k.j.meessen@tue.nl).

Color versions of one or more of the figures in this paper are available online at http://ieeexplore.ieee.org.

Digital Object Identifier 10.1109/TMAG.2010.2042038

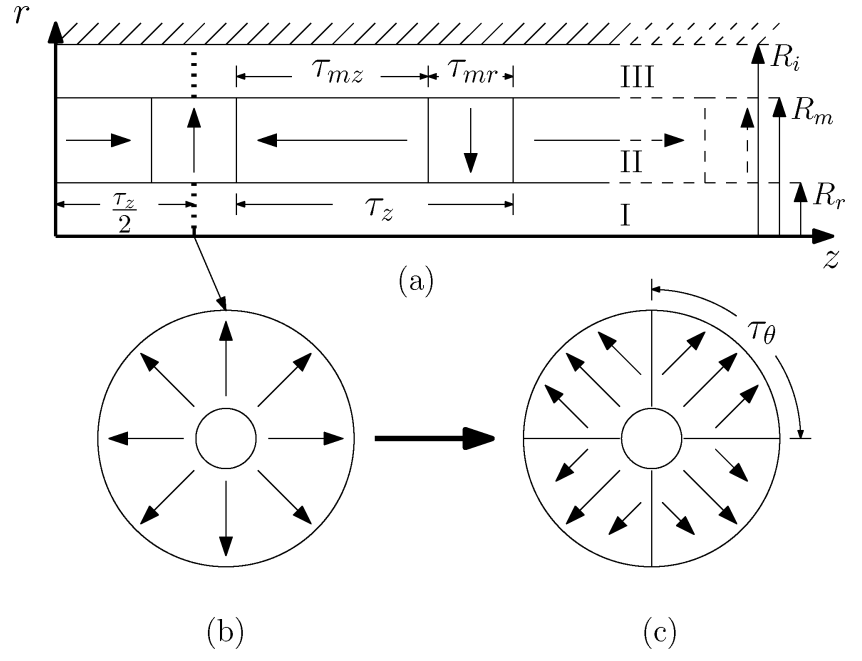

Fig. 1. (a) Cylindrical quasi-Halbach magnet array, (b) ideal radial magnetized ring magnet, (c) approximated radial magnetized ring magnet.

calculate the 3-D field effects. In Section II the derivation of the model including the analytical solution for the magnetic scalar potential and the flux density is presented. Section III compares the results from the model with finite element simulations.

\section{ModELING}

The aim of the model is to calculate the magnetic field in region I, II and III as depicted in Fig. 1(a). To obtain a model describing the magnetic fields, the Maxwell equations can be solved using the magnetic vector potential $\vec{A}$ or the magnetic scalar potential $\varphi$. The magnetic vector potential results in a description of the magnetic flux density $\vec{B}$ while the magnetic scalar potential gives the magnetic field strength $\vec{H}$.

The main disadvantage of $\varphi$ is that it can be applied only in current free problems, however, this paper investigates only the magnetic field due to the permanent magnets, and hence, the model is current free. A major advantage of $\varphi$ is the reduced complexity in 3-D problems. The magnetic vector potential will result in a vector with three components each a function of $r, z, \theta$, while the magnetic scalar potential is a single scalar function of $r, z, \theta$. Therefore, the magnetic scalar potential is used which is defined as

$$
\vec{H}=-\nabla \varphi .
$$


In the model, the following assumptions are made:

1) The soft-magnetic parts are infinitely permeable

2) The cylinder is infinitely long, the end-effects are not taken into account.

3) The permanent magnets have a linear demagnetization characteristic, and are fully magnetized in the direction of magnetization.

4) All regions are non-conducting and current-free.

The scalar potential has to be solved in the source free regions, I and III, and in the permanent magnet region II. In the latter region this results in the Poisson (2), and in region I and III in the Laplace (3)

$$
\begin{aligned}
\nabla^{2} \varphi & =\frac{1}{\mu_{r}} \nabla \cdot \vec{M} \\
\nabla^{2} \varphi & =0
\end{aligned}
$$

where $\mu_{r}$ is the relative permeability of the permanent magnets and $\vec{M}$ the magnetization vector describing the magnet array by means of a Fourier series.

\section{A. Magnetization}

The magnetization vector describing the cylindrical Halbach array has three components

$$
\vec{M}(\theta, z)=M_{r} \vec{e}_{r}+M_{\theta} \vec{e}_{\theta}+M_{z} \vec{e}_{z}
$$

where $\vec{e}_{r}, \vec{e}_{\theta}, \vec{e}_{z}$ are the unit vectors in the radial, angular and axial direction respectively. The position dependency is provided by the product of two Fourier series. The first Fourier series describes the magnetization as function of $\theta$ and has a fundamental period of $2 \tau_{\theta}$. This pole pitch $\tau_{\theta}$ is defined by the number of segments of the ring magnet, $N_{s}$, through $\tau_{\theta}=$ $(2 \pi) /\left(N_{s}\right)$. The second Fourier series describes the dependency in the $z$ direction and has a fundamental period of $2 \tau_{z}$.

In general, the magnetization of a quasi-Halbach array can be split in two parts, the normal magnetized magnets and the tangential magnetized magnets.

1) Normal Magnetized Magnets: The normal magnetized magnets are in this case the segmented diametrically magnetized ring magnet as shown in Fig. 1(c). Due to these diametrically magnetized magnets, the magnetization vector contains, besides a radial component, $M_{r}$, a component in the angular, direction, $M_{\theta}$. In Fig. 2, the two components of the magnetization are shown representing a magnet which consists of four segments.

Besides the dependency of $\theta, M_{r}$ and $M_{\theta}$ are a function of $z$ as the normal magnetized magnets are enclosed by tangential magnetized magnets in the axial, $z$, direction. Hence, the magnetization can be described as

$$
\begin{aligned}
M_{r}(\theta, z)= & \frac{B_{r e m}}{\mu_{0}} \sum_{n=0}^{\infty} M_{r n} \cos (w \theta) \\
& \times \sum_{k=1}^{\infty} M_{r k} \sin (m z) \\
M_{\theta}(\theta, z)= & \frac{B_{r e m}}{\mu_{0}} \sum_{n=0}^{\infty} M_{\theta n} \sin (w \theta) \\
& \times \sum_{k=1}^{\infty} M_{\theta k} \sin (m z)
\end{aligned}
$$
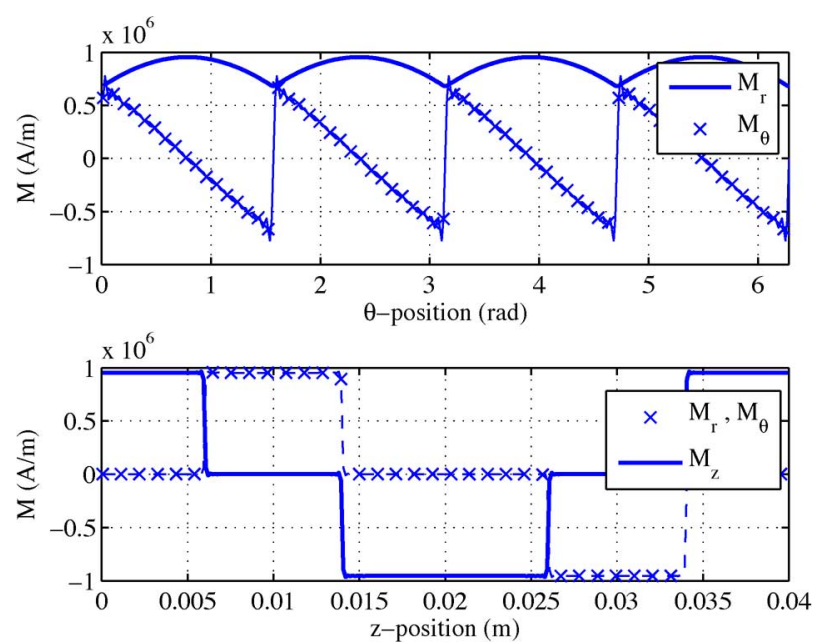

Fig. 2. Magnetization vector where the upper graph describes a ring magnet containing four segments as function of $\theta$ and the second graph describes the magnetization as function of $z$

where $w=(n \pi) /\left(\tau_{\theta}\right)$, are the spatial frequencies in the angular direction and $m=(k \pi) /\left(\tau_{z}\right)$, are the spatial frequencies in the axial direction. Since $M_{r}$ has a DC-offset in the angular direction, as can be seen in Fig. 2, the summation of the Fourier series describing the angular dependency starts from $n=0$. The coefficients of the Fourier series are

$$
\begin{aligned}
& M_{r n}= \begin{cases}\frac{-8 \pi \cos \left(\frac{n \pi}{2}\right)^{2}(-1)^{n} \sin \left(\frac{\pi}{N_{s}}\right)}{N_{s}\left(n^{2} \pi^{2}-\left(\frac{2 \pi}{N_{s}}\right)^{2}\right)} & \text { for } n \neq \frac{2}{N_{s}} \\
0 & \text { for } n=\frac{2}{N_{s}} \\
\frac{N_{s} \sin \left(\frac{\pi}{N_{s}}\right)}{\pi} & \text { for } n=0\end{cases} \\
& M_{r k}=\frac{4}{k \pi} \sin \left(\frac{\left(1-\frac{\tau_{m r}}{\tau_{z}}\right) k \pi}{2}\right) \sin \left(\frac{k \pi}{2}\right) \\
& M_{\theta n}= \begin{cases}\frac{4 n \pi \cos \left(\frac{n \pi}{2}\right)^{2} \sin \left(\frac{\pi}{N_{s}}\right)}{n^{2} \pi^{2}-\left(\frac{2 \pi}{N_{s}}\right)^{2}} & \text { for } n \neq \frac{2}{N_{s}} \\
0 & \text { for } n=\frac{2}{N_{s}} \\
0 & \text { for } n=0\end{cases} \\
& M_{\theta k}=M_{r k} \text {. }
\end{aligned}
$$

2) Tangential Magnetized Magnets: To reduce the complexity of the expression of the magnetic scalar potential, the magnetization of the tangential (or axially) magnetized magnets, $M_{z}$, has the same form as $M_{r}$ and $M_{\theta}$

$$
M_{z}(\theta, z)=\frac{B_{r e m}}{\mu_{0}} \sum_{n=0}^{\infty} M_{z n} \cos (w \theta) \sum_{k=1}^{\infty} M_{z k} \cos (m z) .
$$

However, $M_{z}(\theta, z)$ is independent of the angular position, $\theta$, as described in Fig. 2. Therefore, $M_{z n}$ has only a nonzero value for $n=0$, while $M_{z k}$ describes a square wave.

$$
\begin{aligned}
& M_{z n}= \begin{cases}0 & \text { for } n \neq 0 \\
1 & \text { for } n=0\end{cases} \\
& M_{z k}=\frac{4}{k \pi} \cos \left(\frac{\left(1-\alpha_{p z}\right) k \pi}{2}\right) \sin \left(\frac{k \pi}{2}\right)
\end{aligned}
$$




\section{B. Magnetic Field Description}

In [4] a solution for the magnetic scalar potential for a 3-D problem with radial magnetization in cylindrical coordinates is given. The solution consists of a Fourier series where the coefficients contain modified Bessel functions of the first and second kind, $\mathcal{I}_{w}(m r)$ and $\mathcal{K}_{w}(m r)$ respectively. For the problem described in this paper, a similar solution is used, however the magnetization in this problem has, besides a radial component, components in the $z$ and $\theta$ direction.

Solving (2) using the method of separation of variables results in the magnetic scalar potential as given in (14). Inserting this expression in (1) results in expressions for the three components of the magnetic field with unknown coefficients $a_{n k}$ and $b_{n k}$ for each region. These equations describe the magnetic field in all regions, I, II and III, as shown in Fig. 1. In the source free regions I and III, the expression can be simplified because the functions $G_{r}, G_{z}, G_{\theta}$ are zero

$$
\begin{aligned}
& \varphi(r, \theta, z)=\sum_{n=0}^{\infty} \sum_{k=1}^{\infty} \\
& -\frac{1}{m}\left(a_{n k} \mathcal{I}_{w}(m r)+b_{n k} \mathcal{K}_{w}(m r)\right. \\
& \left.+G_{r}(r)+G_{z}(r)+G_{\theta}(r)\right) \cos (w \theta) \sin (m z) \\
& G_{r}(r)=\frac{m M_{r k} M_{r n} B_{r e m}}{\mu_{0} \mu_{r}} \\
& \times\left(\mathcal{I}_{w}(m r) \int_{R_{r}}^{r} \mathcal{K}_{w}(m \nu) d \nu\right. \\
& \left.-\mathcal{K}_{w}(m r) \int_{R_{r}}^{r} \mathcal{I}_{w}(m \nu) d \nu\right) \\
& G_{\theta}(r)=-\frac{w m M_{\theta k} M_{\theta n} B_{r e m}}{\mu_{0} \mu_{r}} \\
& \times\left(\mathcal{I}_{w}(m r) \int_{R_{r}}^{r} \nu \mathcal{K}_{w}(m \nu) d \nu\right. \\
& \left.-\mathcal{K}_{w}(m r) \int_{R_{r}}^{r} \nu \mathcal{I}_{w}(m \nu) d \nu\right) \\
& G_{z}(r)=\frac{m m M_{z k} M_{z n} B_{r e m}}{\mu_{0} \mu_{r}} \\
& \times\left(\mathcal{I}_{w}(m r) \int_{R_{r}}^{r} \mathcal{K}_{w}(m \nu) d \nu\right. \\
& \left.-\mathcal{K}_{w}(m r) \int_{R_{r}}^{r} \mathcal{I}_{w}(m \nu) d \nu\right) \text {. }
\end{aligned}
$$

\section{Boundary Conditions}

The unknown coefficients $a_{n k}$ and $b_{n k}$ for each region in (14) can be found by solving boundary conditions in all regions. From the axis-symmetric coordinate system follows the first Dirichlet boundary condition. Furthermore, the magnetic scalar potential, $\varphi$, and the normal component of the magnetic flux density, $B_{r}$, should be continuous at each interface resulting in boundary condition 2 to 5 as listed below. As the iron at $r=R_{i}$ is assumed to be infinitely permeable, the tangential components of the magnetic field strength in region III at $r=R_{i}$ should be
TABLE I

MODEL PARAMETERS

\begin{tabular}{lll}
\hline Property & Value & Description \\
\hline$B_{\text {rem }}(\mathrm{T})$ & 1.2 & Remanent flux density of PM \\
$\mu_{r}$ & 1.05 & Relative permeability of PM \\
$R_{r}(\mathrm{~mm})$ & 4.0 & Magnet inner radius \\
$R_{m}(\mathrm{~mm})$ & 8.0 & Magnet outer radius \\
$R_{i}(\mathrm{~mm})$ & 10.0 & Iron inner radius \\
$\tau_{z}(\mathrm{~mm})$ & 20.0 & Pole pitch in axial direction \\
$\alpha_{p z}$ & 0.6 & Magnet to pole pitch ratio \\
$N_{s}$ & 4 & Number of segments of ring magnet \\
$\tau_{\theta}(\mathrm{mm})$ & $\frac{\pi}{2}$ & Pole pitch in angular direction \\
$N$ & 20 & Number of harmonics in angular direction \\
$K$ & 20 & Number of harmonics in axial direction \\
\hline
\end{tabular}

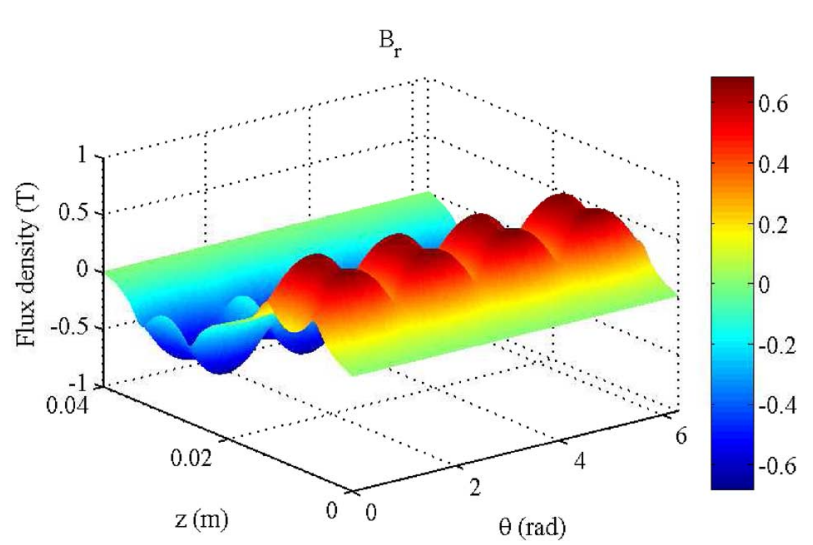

Fig. 3. A 3-D representation of the radial component of the flux density in the center of region III, calculated using the semi-analytical model.

zero. As the magnetic field strength is the derivative of the magnetic scalar potential (14), the magnetic scalar potential should be constant, $c$, at this radius
1) $\varphi_{I}=\left.0\right|_{r=0} \forall \quad z, \theta$
2) $\varphi_{I}=\left.\varphi_{I I}\right|_{r=R_{r}} \forall \quad z, \theta$
3) $B_{r I}=\left.B_{r I I}\right|_{r=R_{r}} \forall z, \theta$
4) $\varphi_{I I}=\left.\varphi_{I I I}\right|_{r=R_{m}} \forall z, \theta$
5) $B_{r I I}=\left.B_{r I I I}\right|_{r=R_{m}} \forall z, \theta$
6) $\varphi_{I I I}=\left.c\right|_{r=R_{i}} \forall \quad z, \theta$.

\section{RESULTS}

As the expression of the magnetic scalar potential (14) contains twice an infinite sum, an approximation over a finite number of harmonics is needed to find a solution. Therefore, in the following sections, the number of harmonics describing the potential and magnetic fields in the axial direction is limited to $K$ while the number of harmonics in the angular direction is limited to $N$. Consequently, solving the boundary conditions results in $6 \times K \times N$ equations.

This set of equations is implemented in MATLAB ${ }^{\circledR}$ together with the magnetization and numerical approximations for the integrals in $G_{r}(r), G_{z}(r), G_{\theta}(r)$. This results in a solution for the magnetic fields in the three regions as shown in Fig. 1(a). The magnetic field distribution is calculated for the geometric parameters given in Table I. In Fig. 3, the radial component of the flux density in the center of region III is shown. The four segments of the normal magnetized magnet are clearly visible. 

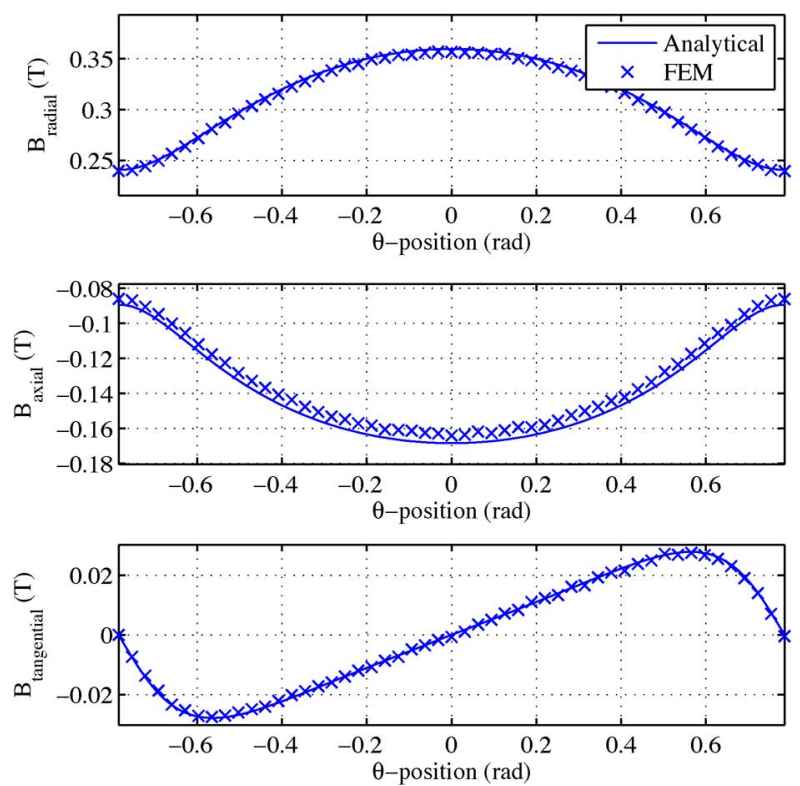

Fig. 4. Comparison of the flux density in the center of region III (at $r=9.0$ $\mathrm{mm}$ ) calculated by FEM model and the analytical model. The three components of the flux density are shown as function of the angular position at a constant $z=5.0 \mathrm{~mm}$.

\section{A. Comparison With FEM}

The results of the model are verified with a 3-D FEM model created in FLUX3D with the same assumptions as listed in Section II, i.e., an infinitely long cylinder and infinitely permeable iron. The magnetic field distribution in the center of region III at $r=9.0 \mathrm{~mm}$ is depicted in Figs. 5 and 4. In Fig. 5, the three components of the flux density are calculated for a constant $z=5.0 \mathrm{~mm}$ as function of the angular position $\theta$. The figure shows the flux density for $-\left(\tau_{\theta}\right) /(2)<\theta<\left(\tau_{\theta}\right) /(2)$ and shows good agreement between the FEM model and the analytical model. Fig. 4 shows the flux density components for a constant $\theta=-(\pi) /(8)$ as function of the axial position for $-\left(\tau_{z}\right) /(2)<z<\left(\tau_{z}\right) /(2)$. The deviation between the two models is in all points smaller than $3 \%$. However, as can be seen for the tangential component of the flux density in Fig. 4, the FEM results contain some small fluctuations, which contribute to the total deviation as well.

\section{CONCLUSION}

This paper concerns the 3-D modeling of a cylindrical quasi-Halbach permanent magnet array. The effects of segmentation of the normal magnetized magnets are calculated
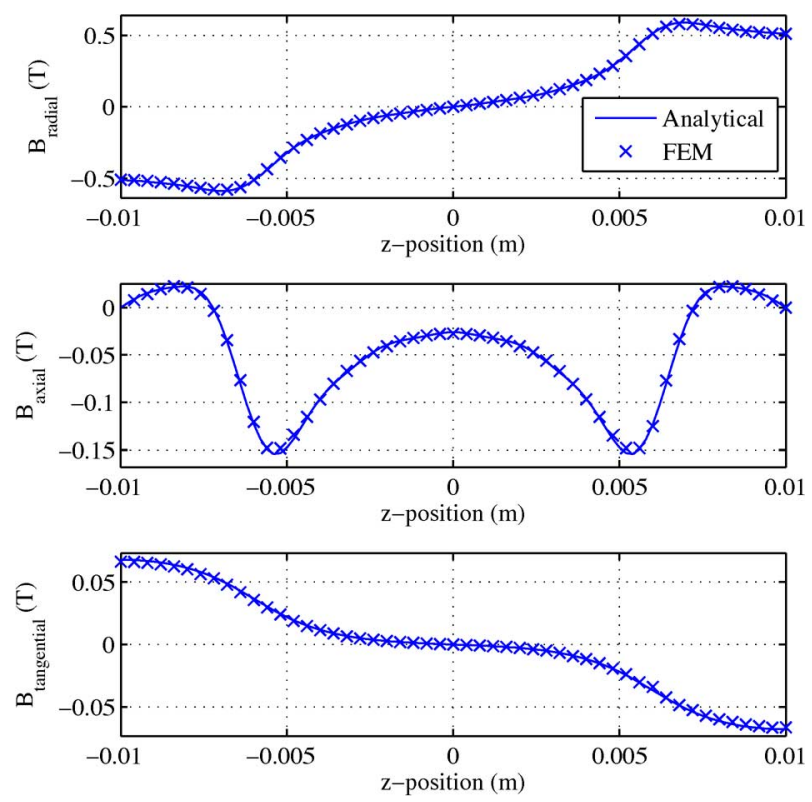

Fig. 5. Comparison of the flux density in the center of region III (at $r=9.0$ $\mathrm{mm}$ ) calculated by FEM model and the analytical model. The three components of the flux density are shown as function of the axial position at a constant $\theta=$ $-(\pi) /(8) \mathrm{rad}$.

which were neglected in previous publications regarding 2-D modeling quasi-Halbach arrays [2], [3]. The derivation of a three dimensional semi-analytical model describing the segmentation effect in a quasi-Halbach cylinder is presented. The magnetic field description is obtained by solving the Maxwell equations using the magnetic scalar potential. The resulting model is implemented and verified with a FEM model and shows good agreement. The model can be used to calculate the effect of using segments in a quasi-Halbach array without the need for a time-consuming FEM analysis.

\section{REFERENCES}

[1] K. Halbach, "Design of permanent multipole magnets with oriented rare earth cobalt material," Nucl. Instr. Methods, vol. 69, no. 1, pp. $1-10,1980$.

[2] W.-J. Kim, M. Berhan, D. Trumper, and J. Lang, "Analysis and implementation of a tubular motor with Halbach magnet array," in Proc. Thirty-First IAS Conf., Oct. 1996, vol. 1, pp. 471-478, vol. 1.

[3] J. Wang and D. Howe, "Tubular modular permanent-magnet machines equipped with quasi-Halbach magnetized magnets-Part I: Magnetic field distribution, EMF, and thrust force," IEEE Trans. Magn., vol. 41, no. 9, pp. 2470-2478, Sep. 2005.

[4] A. Youmssi, "A three-dimensional semi-analytical study of the magnetic field excitation in a radial surface permanent-magnet synchronous motor,' IEEE Trans. Magn., vol. 42, no. 12, pp. 3832-3841, Dec. 2006. 